\title{
La investigación sobre los mensajes comunicacionales en México: 1990-2002
}

\section{José Carlos Lozano}

Centro de Investigación en Comunicación e Información

Tecnológico de Monterrey, Campus Monterrey

64849 Monterrey, Nuevo León, México

jclozano@itesm.mx

\section{Resumen}

El artículo ofrece una revisión de la investigación mexicana reciente sobre mensajes comunicacionales publicada en las revistas académicas más importantes de ese país de 1990 a 2002. El diagnóstico discute las principales líneas de investigación y enfoques teóricos utilizados en este campo y concluye con una evaluación de las fortalezas, deficiencias y retos para la investigación actual y futura de los contenidos comunicacionales.

Se analiza un total de treinta y cinco análisis de contenido y se presenta una panorámica general de los énfasis conceptuales y temáticos dominantes en el campo. El trabajo señala que el tipo de estudios más abundante en el período de trece años fue el relativo al flujo y oferta de programación televisiva, es decir, reportes de investigación que no se centraban propiamente en las características del contenido, sino en el número particular de mensajes en las parrillas programáticas de la televisión mexicana, según su origen, género y tiempo de transmisión. En segundo término sobresalían los estudios sobre noticias internacionales inspirados por el NOMIC, y en tercer lugar trabajos sobre rutinas productivas y valores profesionales implícitos en el contenido de los mensajes.

Palabras clave: Análisis de contenido, mensajes comunicacionales, investigación mexicana de la comunicación, sociología de la producción de noticias, establecimiento de la agenda, estereotipos, NOMIC.

\section{Abstract. Content analysis as a research technique in Mexican communication research: 1990-2002}

This paper presents a review of current Mexican research on media messages published in the major academic journals and books in communication in Mexico from 1990 to 2002. The review provides an overview of the principal research lines and theoretical approaches used in this field, and concludes with an evaluation of the strengths, deficiencies, and challenges for current and future research.

Thirty-five Mexican content analysis reports are reviewed, and a general overview of the conceptual and thematic emphases dominant in the field is presented. The paper points out that the most abundant type of studies in the 13-year period reviewed were the ones about the flow and supply of television programming, in other words, studies not properly about the characteristics of the content but about the number of particular messages in Mexican TV according to their origin, genre, and time of transmission. Next are studies 
about international news inspired by the NWICO, and then content analysis of media coverage of presidential elections, and studies about media routines and professional values.

Key words: Content Analysis, media messages, Mexican communication research, Newsmaking, Agenda Setting, Stereotypes, NWICO.

\section{Sumario}

Escasez de investigaciones basadas en análisis de contenido

Análisis de contenido sobre condicionantes individuales, rutinas productivas y valores profesionales Cobertura de las elecciones presidenciales
Ofertas programáticas y Nuevo Orden Mundial de la Información

Análisis de estereotipos y violencia en la ficción televisiva y cinematográfica Conclusiones

Tradicionalmente, la investigación mexicana sobre el contenido de los mensajes comunicacionales ha sido significativamente menor a los estudios sobre la economía política de los medios y los análisis de audiencias. Los académicos nacionales, inscritos inicialmente en las perspectivas de la teoría de la dependencia y del imperialismo cultural y posteriormente en los enfoques de estudios culturales, han privilegiado las discusiones sobre los condicionantes y las vinculaciones de los conglomerados mediáticos con las elites económicas y políticas, o las mediaciones y las lecturas que realizan al exponerse a los medios.

El estudio de los mensajes, así, a pesar de su relevancia para ambos tipos de investigaciones, ha quedado permanentemente en un segundo plano. Las múltiples afirmaciones de la economía política sobre la influencia de propietarios, accionistas, anunciantes y funcionarios públicos en el contenido de los mensajes, por lo tanto, no han sido corroboradas sistemáticamente con análisis cuantitativos o cualitativos. Y algo similar ha ocurrido con las investigaciones sobre audiencias, que por no tomar en cuenta los tipos de significados preferentes o alternativos en los mensajes, se han limitado a documentar aspectos generales de los usos de los medios y su integración en la vida cotidiana, evadiendo el problema central de las lecturas preferentes, negociadas o hegemónicas.

El presente artículo ofrece un diagnóstico sintético sobre el estado actual de las investigaciones mexicanas sobre los mensajes comunicacionales publicadas en las principales revistas y publicaciones científicas del país ${ }^{1}$. Se ofrece

1. Solamente se incluyen los artículos, libros o capítulos de libros publicados en revistas científicas mexicanas. No se toman en cuenta tesis de licenciatura, maestría o doctorado, ni reportes de investigación publicados en revistas no reconocidas como científicas. Solamente se seleccionaron reportes de investigaciones empíricas basadas en la técnica del análisis de contenido cuantitativo y publicados de 1990 a la fecha. Ensayos o tratados metodológicos sobre la técnica del análisis de contenido también fueron excluidos del trabajo. 
una panorámica sobre las principales líneas de investigación, enfoques teóricos y metodologías utilizadas en este campo y se concluye con una evaluación de los avances logrados y las limitaciones y retos pendientes.

\section{Escasez de investigaciones basadas en análisis de contenido}

Una revisión de las ediciones de las revistas y libros mexicanos de comunicación más importantes de 1990 a la fecha muestra una escasez significativa de estudios basados en la técnica del análisis de contenido.

La cantidad de treinta y cinco trabajos empíricos publicados en revistas como el Anuario de Investigación de la Comunicación CONEICC, Comunicación y Sociedad, Espacios de Comunicación, Estudios sobre las Culturas Contemporáneas y Revista Mexicana de Comunicación, así como diversas antologías y libros, indica el bajo uso de una de las técnicas más accesibles y útiles para documentar el contenido de los mensajes comunicacionales: tres reportes por año. Si consideramos que los análisis cualitativos son aún más escasos, podemos percatarnos claramente de la existencia de enormes lagunas de información sobre el contenido de los mensajes.

El cuadro 1 muestra que en 1995 y 1996 hubo un pico de diez trabajos publicados (cinco por año), pero a partir de ahí la producción bajó a dos por año, excepto para el 2000, donde subió a siete, para bajar de nuevo a dos en el 2001. Los datos, así, indican que, más que observarse una tendencia sistemática al aumento del uso de esta técnica, se dan concentraciones esporádicas en ciertos años por la existencia de proyectos específicos (como el Monarca en 1995-1996) o acontecimientos coyunturales, como las elecciones presidenciales de 1988 y del 2000.

¿Cuáles son las razones para este panorama, que es preocupante no por el rechazo a la técnica de investigación en cuestión, sino por la carencia que implica de descripciones y análisis de los contenidos comunicacionales mexicanos? Una respuesta parece encontrarse en el predominio de posturas teóricas que aún en estas fechas de tolerancia y promoción de la triangulación metodológica descalifican el uso de técnicas cuantitativas por considerarlas inherentemente positivistas. Otra razón puede estar relacionada con una tendencia hacia el ensayismo en menoscabo de los estudios empíricos, tendencia largamente prevaleciente en los trabajos mexicanos (y en buena medida latinoamericanos) sobre comunicación. Una tercera, relacionada con la anterior, podría basarse en el desconocimiento de los métodos e instrumentos empíricos que involucra esta técnica entre los docentes y los académicos. Y por último, una cuarta razón pareciera encontrarse en el auge de modas teóricas y metodológicas que en los últimos años han propiciado que la mayoría de las investigaciones empíricas se concentren en estudios sobre la recepción y el consumo por parte de las audiencias mexicanas.

Por las razones que sea, lo que resulta evidente es la dificultad de los análisis económico-políticos de documentar y comprobar sus afirmaciones sobre el impacto de los condicionantes estructurales en la producción y contenido 
de los mensajes de los conglomerados de medios. Asimismo, esta escasez de datos sobre los significados comunicacionales impide que los partidarios de los estudios culturales puedan concentrarse en el análisis del tipo de lecturas (preferentes, negociadas u oposicionales) realizadas por los diferentes segmentos o grupos culturales que componen la audiencia. Esto, que resulta evidente tan sólo al evaluar la cantidad de trabajos enfocados al análisis de los contenidos comunicacionales, será confirmado en las siguientes secciones, al detallar el tipo de datos y enfoques prevalecientes en las pocas investigaciones basadas en esta técnica.

\section{Análisis de contenido sobre condicionantes individuales, rutinas productivas $\mathrm{y}$ valores profesionales}

Dentro de los análisis de contenido que se apoyan explícitamente en enfoques teóricos, sobresalen los relativos a identificar en los contenidos noticiosos evidencias de las rutinas productivas y los valores profesionales en operación al ser producidos. Por lo menos siete de los estudios tomados en cuenta discuten en su introducción teórica elementos de los enfoques del newsmaking o sociología de la producción de noticias. Algunos analizan las rutinas de trabajo de los reporteros al cubrir acontecimientos extraordinarios que evidencian sesgos y omisiones. Fregoso (1992/1993), por ejemplo, detectó la ausencia de ingredientes explicativos y contextuales en la cobertura periodística local de las explosiones de hidrocarburos en un colector de la ciudad de Guadalajara, que dejaron un saldo de doscientos muertos y trece kilómetros de calles arrasadas. Lozano (1993), por su parte, estudió la cobertura periodística de una manifestación cívica en la ciudad fronteriza de Nuevo Laredo que terminó en actos vandálicos contra las oficinas de la aduana federal junto al puente internacional que permite el paso a Laredo, Texas, Estados Unidos. Al encontrar que el periódico regiomontano El Norte, el más importante del noreste de México, había favorecido claramente las versiones oficiales que condenaban los actos vandálicos sin balancearlas con las de los manifestantes y líderes involucrados (como lo exigía su propio manual de estilo en casos de controversia), Lozano infirió que los procesos rutinizados de recogida de datos y la internalización de valores profesionales en los reporteros y jefes de sección o de información favorables a las fuentes gubernamentales, eran los principales causantes de una cobertura sesgada hacia la versión oficial.

Otros de los trabajos basados en los enfoques de sociología de la producción noticiosa, a diferencia de los anteriores, se concentran en estudiar el flujo rutinario de noticias sensacionalistas (Cervantes, 1996a; Orellana, 1997) o de noticias nacionales en la prensa mexicana (Arellano et al., 1995; Agudelo, 1996; Cervantes, 1996b; Lozano, 1994). En el caso de los primeros, se trata de análisis de contenido de la transmisión de noticias policíacas en noticieros televisivos (Cervantes, 1996a) o de diarios vespertinos (Orellano, 1997), lo que constituye una línea novedosa de investigación en el ámbito mexicano. 
Cervantes, después de analizar el noticiero regional Al Tanto, de Guadalajara, discute los valores noticiosos que aparecen en el plano de los mensajes y que, según concluye, se relacionan principalmente con el «macrovalor» relacionado con la «satisfacción de las necesidades de la organización» (p. 134). Orellano, por su parte, se concentra en el análisis de las características sociodemográficas de los protagonistas de la información policíaca, y concluye que las rutinas de trabajo y los valores profesionales de los periodistas propician que éstos ignoren el contexto social e histórico que produce y condiciona la existencia de los delitos, para enfocarse en el delincuente, el individuo transgresor que se desvía de la norma social.

Los cuatro trabajos sobre el flujo rutinario de noticias nacionales en periódicos diarios, por su parte, coinciden en demostrar el centralismo en el origen y procedencia de las noticias nacionales (el Distrito Federal), la dependencia de los medios informativos medianos y pequeños en agencias informativas y no en corresponsales o enviados propios, y la reducida agenda de temas propuestos por los diarios a las audiencias mexicanas, en los que sobresalen la política, la administración pública y la economía y se omiten otros igual de relevantes como el medio ambiente, la educación, la salud y la seguridad pública (cfr. Arellano et al., 1995; Agudelo, 1996; Cervantes, 1996b; Lozano, 1994).

Las lagunas en esta área de la sociología de la producción de noticias, sin embargo, son muchas. Por un lado, faltan análisis de contenido de los principales diarios y noticieros televisivos nacionales que documenten variables relativas a las influencias individuales susceptibles de ser detectadas en las noticias, como el sexo y la edad de los reporteros y conductores. Por otro lado, se requieren diagnósticos de las fuentes informativas utilizadas por dichos medios, para definir si las rutinas productivas y los condicionantes organizacionales propician el privilegiar a los actores oficiales o gubernamentales. En general, la escasez de investigadores y de estudios centrados en el análisis de las influencias individuales, organizacionales y productivas al interior de los medios informativos en México, se reproducen en la investigación de los mensajes comunicacionales, a pesar de su prominencia en otros países del mundo.

\section{Cobertura de las elecciones presidenciales}

Esporádicamente, en tiempos de elecciones presidenciales, proliferan análisis de contenido de su cobertura. Por un buen tiempo, dichos estudios se enfocaron exclusivamente a medir el tiempo o el espacio concedidos al partido o candidato oficial frente a los de oposición, así como la tendencia positiva o negativa de las informaciones, dadas las condiciones de control, autocensura y orientación gubernamental de los medios informativos. Este fue el caso de las elecciones de 1988, las que generaron diversos trabajos, entre los que sobresalieron los de Arredondo (1991), Fregoso (1991) y Trejo Delarbre (1991a, $1991 b$ ). De hecho, las tres obras forman parte de un mismo proyecto, basado en un análisis de contenido riguroso y exhaustivo del espacio o tiempo con- 
cedido a cada partido y candidato en las elecciones de ese año, así como de la tendencia en la cobertura según el candidato o partido. Los hallazgos documentan lo que era esperable dadas las circunstancias del sistema político mexicano y su relación con los medios en esos años: un marcado favoritismo cuantitativo y cualitativo hacia el PRI y su candidato Salinas de Gortari, y un vacío de información, o ángulos negativos, sobre el resto de los candidatos.

En las elecciones recientes, donde triunfara por primera vez un candidato de oposición, la tendencia continuó en análisis de contenido destinados a medir el acceso proporcionado a los distintos candidatos y partidos (cfr. Acosta y García, 2000; Toussaint, 2000), pero empezaron a surgir trabajos donde además de lo anterior se determinaba la concentración de las noticias electorales en temas de espectacularización, personalización y dramatización en detrimento de los relacionados con las plataformas electorales o los programas de gobierno de los candidatos (Lozano, 2001; Lozano, García, López, Medina, Mendé, Smith y Solís, 2001). Estos trabajos, a diferencia de los de 1988 , concluyeron que en lo cuantitativo se había dado un mayor balance en la cobertura de los distintos candidatos y partidos (contrastante de acuerdo al medio informativo en cuestión) y que ante la nueva libertad de expresión propiciada por el debilitamiento del régimen priísta y las transformaciones del sistema político mexicano, los medios comerciales se habían orientado radicalmente a coberturas espectaculares y sensacionalistas del proceso electoral.

Además del número tan bajo de investigaciones sobre estos procesos claramente trascendentales y necesarios para la contrastación empírica de teorías y enfoques como el establecimiento de la agenda, la espiral del silencio y la economía política crítica, llama la atención la ausencia notoria de estudios sobre otros procesos electorales y, más allá de ellos, sobre la cobertura de la gestión gubernamental, los informes presidenciales y muchos otros temas relacionados con la comunicación política. A diferencia del apartado anterior, donde la escasez de estudios era similar en el interior de las organizaciones de medios y en el análisis de contenido de las noticias, aquí se presenta un caso muy distinto. Los ensayos y análisis económico-políticos de los procesos electorales tienden a ser numerosos, pero no así los estudios empíricos de sus coberturas mediáticas. Se echan de menos, asimismo, trabajos basados en los enfoques del establecimiento de la agenda y de la espiral del silencio, entre otros.

\section{Ofertas programáticas y Nuevo Orden Mundial de la Información}

Sin duda los diagnósticos de la oferta programática televisiva mexicana han sido numerosos y permanentes a partir de la década de los noventa. Aunque no son propiamente análisis de contenido, porque se dedican exclusivamente a clasificar los contenidos por géneros, origen y horarios, han permitido documentar con cierta sistematicidad y detalle las características de la oferta que se les presenta a las audiencias de la televisión abierta nacional. 
La mayoría de los trabajos en este apartado tienen un interés particular en documentar la disponibilidad de contenidos nacionales en comparación con los extranjeros (Crovi y Vilar, 1995; Lozano, 1995-1996, 1998, 2000; Lozano y García Núñez de Cáceres, 1995; Sánchez Ruiz, 1995, 1996), para definir si se da el fenómeno de imperialismo cultural o de interdependencia asimétrica en los flujos globales de programación en el caso mexicano. Los resultados, en la gran mayoría de los casos, han coincidido en señalar un predominio de contenidos importados en el género de la ficción (películas y series), pero una hegemonía de las producciones nacionales en los géneros informativos, los deportes y los programas de variedades y concursos.

A pesar del auge relativo de esta línea de investigación, es claro que se concentra en los años de 1995 y 1996 gracias a los esfuerzos del Proyecto Monarca entre investigadores canadienses y mexicanos. En los últimos años, por ejemplo, se observa una disminución radical en el número de estos trabajos, con la excepción de un diagnóstico de oferta enfocado más bien al ámbito nacional (Guadarrama, 1999), lo que deja sin continuidad y consolidación este tipo de análisis.

En este mismo campo de preocupaciones sobre el NOMIC y los equilibrios en los flujos comunicacionales se encuentran los análisis de la información internacional en la prensa mexicana y latinoamericana. Centrados, aquí sí, en las características específicas de las fuentes y temáticas de las noticias extranjeras, así como en el equilibrio o desbalance de informaciones por regiones y países del mundo (Huerta, 2000; Lozano, 2000; Pérez, 1997), estos análisis permiten explorar la validez de las acusaciones del NOMIC sobre el favorecer a los países industrializados tanto cuantitativa como cualitativamente.

\section{Análisis de estereotipos y violencia en la ficción televisiva y cinematográfica}

Es sin duda en esta línea de la investigación de los contenidos de ficción donde más se extraña la presencia de trabajos empíricos. Durante los doce años tomados en cuenta, apenas aparecen tres publicaciones derivadas de solamente dos estudios sobre contenidos de ficción. Los dos primeros, de Lozano, De la Fuente, Garza y Treviño (1997, 1999), reportan los resultados de un análisis de los estereotipos demográficos, ocupacionales y étnicos en películas de estreno en los videoclubes mexicanos. El tercero, de Quintero y López (1999), muestra los hallazgos de un análisis de contenido sobre los estereotipos femeninos en las telenovelas mexicanas, un tema que en los setenta y principios de los ochenta tuvo muchos seguidores, pero que a partir de los noventa ha quedado relegado significativamente.

Es indiscutible que en este rubro está prácticamente todo por hacer. Se requieren análisis de contenido sistemáticos y amplios sobre la representación y estereotipos de las minorías demográficas, ocupacionales y étnicas en la televisión, el cine, los vídeos, las historietas y demás mensajes comunicacionales 
de ficción. Asimismo, hacen falta investigaciones sobre los contenidos violentos y sobre los valores reflejados en los contenidos televisivos y cinematográficos. Enfoques como el imperialismo cultural y el análisis del cultivo, entre otros, han argumentado convincentemente sobre la importancia crucial de estas representaciones en la socialización y en las visiones del mundo y de la vida de las audiencias, por lo que urge revitalizar esta veta de análisis de contenido en el país.

\section{Conclusiones}

El análisis de contenido cuantitativo es una herramienta metodológica sumamente útil para describir las características de los contenidos mediáticos y para realizar inferencias sobre los procesos de producción y recepción de los mensajes. Su sencillez y su bajo costo la convierten en una técnica ideal para realizar investigaciones sistemáticas y confiables en países como México, donde escasea el financiamiento y el apoyo institucional. Como instrumento empírico de recolección de datos, no está condicionada por una perspectiva conceptual específica, y puede ser y ha sido ampliamente utilizada por enfoques teóricos tan divergentes como los positivistas-funcionalistas y los marxistas o críticos.

Desafortunadamente, el bajo número de investigadores de la comunicación prevaleciente en México, y las condiciones en que realizan sus estudios, han provocado que dependiendo del momento, los temas, métodos y enfoques teóricos varíen año con año. Esto ha obstaculizado, con muy pocas excepciones, la consolidación de líneas de investigación y grupos de interés centrados permanentemente en ellas. A diferencia de otros países, donde determinados investigadores mantienen su atención en temáticas relevantes por años, y donde los académicos se especializan en áreas de la comunicación concretas, en México son pocos quienes logran hacer esto y muchos los que se ven obligados a estar saltando de un tema a otro para responder a la demanda sobre discusión de temas emergentes o coyunturales, del Tratado de Libre Comercio con Estados Unidos y Canadá a los procesos electorales, de las culturas populares a la globalización y las nuevas tecnologías.

Lo anterior ha significado que en México aún existen enormes lagunas de información y análisis en la gran mayoría de las vertientes teóricas. Más que avances sistemáticos e integrales, se presentan por lo general análisis fragmentados en el tiempo y en el espacio que, si bien son muy relevantes para la comprensión de sus objetos de estudio, son claramente insuficientes. Es el caso de enfoques como el newsmaking o sociología de la producción noticiosa, la economía política crítica, o el establecimiento de la agenda, por citar sólo algunos. La situación es mejor en el campo de los estudios culturales, donde la cantidad y calidad de investigaciones desde los ochenta hasta la fecha han permitido mayor consolidación y sistematicidad. Sin embargo, aún en esta corriente se presenta una mayor inclinación hacia las elaboraciones teóricas y las discusiones conceptuales, escaseando las investigaciones empíricas cualitativas 
o cuantitativas de las audiencias y de los significados preferentes o alternativos que les ofrecen los medios.

La revisión de treinta y tres reportes de análisis de contenido mexicanos en este trabajo no es exhaustiva, pero ofrece una panorámica general de los énfasis conceptuales y temáticos prevalecientes en este campo. Sobresalen, en primer lugar, los diagnósticos de la oferta televisiva, los cuales no son propiamente análisis de contenido, sino clasificaciones de los mensajes de acuerdo a su género y procedencia. Le siguen algunos trabajos sobre las características de los mensajes informativos internacionales —inspirados principalmente en el NOMIC_- y algunos en las áreas de comunicación política y elecciones, así como en rutinas productivas.

En este panorama no todo es negativo. Si bien son aún pocos y fragmentados, se observa en los análisis de contenido un uso correcto de la técnica, así como la generación de hallazgos importantes para la comprensión de los fenómenos analizados. El hecho de que sólo cinco de los treinta y tres estudios hayan sido realizados en la Ciudad de México por investigadores capitalinos, demuestra que en la provincia mexicana (principalmente Guadalajara y Monterrey) es donde se está consolidando el uso de esta importante herramienta metodológica.

Falta mucho camino por recorrer. Esperamos que las nuevas condiciones en México de mayor democracia y mayor necesidad de competir ante la globalización y la apertura de fronteras por los tratados comerciales, así como la exigencia de certificaciones y acreditaciones externas a las universidades públicas y privadas, propicie un mayor desarrollo y dinamismo de la investigación comunicacional en México. Y dentro de ésta, el análisis de contenido está llamado a jugar un papel fundamental.

Cuadro 1. Análisis de contenido publicados por año en revistas científicas y libros mexicanos de comunicación

\begin{tabular}{l|c|c|c|c|c}
\hline Año & N.o de trabajos & Año & N.o de trabajos & Año & N.o de trabajos \\
\hline 1990 & 1 & 1994 & 1 & 1998 & 2 \\
\hline 1991 & 4 & 1995 & 5 & 1999 & 3 \\
\hline 1992 & 1 & 1996 & 5 & 2000 & 7 \\
\hline 1993 & 1 & 1997 & 3 & 2001 & 2 \\
\hline
\end{tabular}


Cuadro 2. Sociología de la producción de noticias y estudios de prensa

\begin{tabular}{|c|c|c|}
\hline Fregoso, G. & $1992 / 93$ & $\begin{array}{l}\text { La prensa de Guadalajara: cuatro versiones de una tra- } \\
\text { gedia. }\end{array}$ \\
\hline Lozano, J.C. & 1993 & $\begin{array}{l}\text { Fuentes y versiones gubernamentales en la prensa mexi- } \\
\text { cana: la cobertura del ataque a la aduana de Nuevo } \\
\text { Laredo. }\end{array}$ \\
\hline , J. C. & 1994 & $\begin{array}{l}\text { Tema e fontes na informação nacional em La Jornada, El } \\
\text { Norte e El Porvenir: uma análise da sociologia da orga- } \\
\text { nização dos meios. }\end{array}$ \\
\hline rellano, L. et al. & 1995 & $\begin{array}{l}\text { Temas y fuentes informativas en la prensa mexicana: un } \\
\text { estudio comparativo. }\end{array}$ \\
\hline Aguc & 1996 & $\begin{array}{l}\text { La influencia de las rutinas productivas en la construc- } \\
\text { ción del acontecer nacional: el caso de Siglo } 21 \text {, perió- } \\
\text { dico de Guadalajara, México. }\end{array}$ \\
\hline Cervantes, C. & $1996 a$ & $\begin{array}{l}\text { Especificidades en el análisis de contenido de noticia- } \\
\text { rios de televisión locales. }\end{array}$ \\
\hline Cervantes, C. & $1996 b$ & Valores noticiosos en el periodismo televisivo de nota roja. \\
\hline Orellana, L. & 1997 & $\begin{array}{l}\text { Necrofilia visual: análisis de contenido y de receptores } \\
\text { de La Opinión de la Tarde. }\end{array}$ \\
\hline Inestrosa, $S$. & 2000 & $\begin{array}{l}\text { La agenda noticiosa de la prensa escrita y las iglesias en } \\
\text { México. }\end{array}$ \\
\hline
\end{tabular}

Cuadro 3. Comunicación política: elecciones, agenda, espectacularización y economía política

\begin{tabular}{|c|c|c|}
\hline Fregoso, G. & 1991 & $\begin{array}{l}\text { Análisis sobre información política en la prensa } \\
\text { de Guadalajara: la sucesión presidencial. }\end{array}$ \\
\hline Arredondo, P. & 1991 & $\begin{array}{l}\text { Opacidad en la ventana electrónica: el proceso } \\
\text { electoral de } 1988 \text { en los noticieros televisivos. }\end{array}$ \\
\hline Trejo Delarbre, R. & $1991 a$ & El proceso electoral de 1988. \\
\hline Trejo Delarbre, R. & $1991 b$ & $\begin{array}{l}\text { Campañas y elecciones en la prensa de la Ciudad } \\
\text { de México. }\end{array}$ \\
\hline Acosta, M.; García, V. & 2000 & $\begin{array}{l}\text { La publicidad política por televisión en las elec- } \\
\text { ciones del } 2000 \text { en México. }\end{array}$ \\
\hline Lozano, J.C. & 2001 & $\begin{array}{l}\text { Espectacularización en la cobertura informati- } \\
\text { va de las elecciones mexicanas a la presidencia. }\end{array}$ \\
\hline $\begin{array}{l}\text { Lozano, J.C.; García, H.; } \\
\text { López, G.; Medina, J.; } \\
\text { Mendé, M.B.; Smith, C.; } \\
\text { Solís, M. E. }\end{array}$ & 2001 & $\begin{array}{l}\text { Espectacularización de las elecciones presiden- } \\
\text { ciales. }\end{array}$ \\
\hline Toussaint, F. & 2000 & $\begin{array}{l}\text { Las campañas electorales del } 2000 \text { en televisión: } \\
\text { el caso mexicano. }\end{array}$ \\
\hline
\end{tabular}


Cuadro 4. Nomic, economía política, imperialismo cultural y ofertas programáticas

\begin{tabular}{|c|c|c|}
\hline Crovi, D.; Vilar, J. & 1995 & $\begin{array}{l}\text { Canales abiertos de la Ciudad de México: progra- } \\
\text { mación y preferencias del público. }\end{array}$ \\
\hline $\begin{array}{l}\text { Lozano, J.C.; García } \\
\text { Núñez de Cáceres, J. }\end{array}$ & 1995 & $\begin{array}{l}\text { Oferta de programación audiovisual extranjera en } \\
\text { la televisión aérea de Monterrey, Nuevo León. }\end{array}$ \\
\hline Sánchez Ruiz, E. & 1995 & La agenda televisiva en Guadalajara. \\
\hline Lozano, J.C. & $1995 / 96$ & Oferta y recepción de televisión extranjera en $\mathrm{N}$ \\
\hline $\begin{array}{l}\text { Prozco, G.; } \\
\text { Iiveros, F. }\end{array}$ & 1996 & $\begin{array}{l}\text { La oferta de televisión y su percepción por jóvenes } \\
\text { de la Ciudad de México. }\end{array}$ \\
\hline Ruiz, E. & 1996 & $\begin{array}{l}\text { es y regionales de progra- } \\
\text { de México. }\end{array}$ \\
\hline barr & 1998 & La agenda \\
\hline Lozano, J.C. & 1998 & $\begin{array}{l}\text { Programación en la televisión a } \\
\text { área metropolitana de Monterr }\end{array}$ \\
\hline suadarrama, A. & 1999 & Dinámica familiar y televisión. \\
\hline ozano, J.C. & $2000 \mathrm{a}$ & $\begin{array}{l}\text { Oferta y consumo de contenidos televisivos trans- } \\
\text { nacionales en México. }\end{array}$ \\
\hline , J.C. & 1990 & $\begin{array}{l}\text { Un semestre Salinista en los MMC norteamerica- } \\
\text { nos: análisis de contenido. }\end{array}$ \\
\hline Pérez, M. & 1997 & $\begin{array}{l}\text { El caso de los balseros cubanos desde la óptica del } \\
\text { periódico El Norte, de Monterrey. }\end{array}$ \\
\hline Huerta, J.E. & 2000 & $\begin{array}{l}\text { Análisis de la cobertura de la información interna- } \\
\text { cional en la prensa veracruzana desde la discusión } \\
\text { del Nuevo Orden Mundial de la Información y la } \\
\text { Comunicación. }\end{array}$ \\
\hline Lozano, J.C. et al. & 2000 & International news in the Latin American Press. \\
\hline Lozano, J.C. & $2000 \mathrm{~b}$ & Información internacional y prensa latinoamericana. \\
\hline
\end{tabular}

Cuadro 5. Análisis del cultivo, imperialismo cultural y análisis de ficción

\begin{tabular}{|c|c|c|}
\hline $\begin{array}{l}\text { Lozano, J.C; } \\
\text { De la Fuente, Y.; } \\
\text { Garza, R.; } \\
\text { Treviño, J. }\end{array}$ & 1997 & $\begin{array}{l}\text { Estereotipos demográficos, ocupacionales y raciales } \\
\text { en las películas de estreno de los videoclubes de } \\
\text { Monterrey, Nuevo León. }\end{array}$ \\
\hline $\begin{array}{l}\text { Lozano, J.C.; } \\
\text { De la Fuente, Y.; } \\
\text { Garza, R.; Treviño, J. }\end{array}$ & 1999 & $\begin{array}{l}\text { Imágenes y estereotipos en las películas de estreno } \\
\text { de los videoclubes mexicanos. }\end{array}$ \\
\hline $\begin{array}{l}\text { Quintero, C.; } \\
\text { López, J.R. }\end{array}$ & 1999 & Estereotipos de la mujer en las telenovelas mexicanas. \\
\hline
\end{tabular}




\section{Referencias}

Acosta, M.; García, V. (2000). «La publicidad política por televisión en las elecciones del 2000 en México». Revista Mexicana de Comunicación, 13 (65): 7-11.

Agudelo, I. (1996). "La influencia de las rutinas productivas en la construcción del acontecer nacional: el caso de Siglo 21, periódico de Guadalajara, México». Comunicación y Sociedad, (28): 83-114.

Arellano, L.; Chong, B.; Caña, A.; Gendreau, M.; Guicho, F.; Gutiérrez, M.; Lozano, J.C.; Martínez, F.; Morales, L.; Orellana, L.; Ortiz, M.; Matanzo, L.; Priante, J.A.; QuinTANILla, C.; Román, M.A.; VIllarReal, M.C. (1995). «Temas y fuentes informativas en la prensa mexicana». Anuario de Investigación de la Comunicación CONEICC, 2: 241-259.

ARREDONDO, P. (1991). «Opacidad en la ventana electrónica: el proceso electoral de 1988 en los noticieros televisivos». En: Arredondo, P.; Fregoso, G. y Trejo Delarbre, R. [eds.]. Asi se calló el sistema: comunicación y elecciones en 1988. Guadalajara: Universidad de Guadalajara.

Cervantes, C. (1996a). "Valores noticiosos en el periodismo televisivo de nota roja». Comunicación y Sociedad, 25/26.

- (1996b). «Especificidades en el análisis de contenido de noticiarios de televisión locales». Anuario de Investigación de la Comunicación CONEICC, 3: 77-112.

Crovi, D.; Vilar, J. (1995). "Canales abiertos de la Ciudad de México: programación y preferencias del público». En: CROvI, Delia [coord.]. Desarrollo de las industrias audiovisuales en México y Canadá. México: Facultad de Ciencias Políticas y Sociales, UNAM.

FREGOSO, G. (1991). «Análisis sobre información política en la prensa de Guadalajara: la sucesión presidencial». En: ARREdONDO, P.; Fregoso, G. y Trejo DELARBRE, R. [eds.]. Así se calló el sistema: comunicación y elecciones en 1988. Guadalajara: Universidad de Guadalajara.

- (1992-1993). «La prensa de Guadalajara: cuatro versiones de una tragedia». Comunicación y Sociedad, 16/17.

GuadarRama, A. (1999). Dinámica familiar y televisión. Cuadernos de Investigación de la Universidad Autónoma del Estado de México, 1 (4. ${ }^{\mathrm{a}}$ época).

HuERTA, J.E. (2000). «Análisis de la cobertura de la información internacional en la prensa veracruzana desde la discusión del Nuevo Orden Mundial de la Información y la Comunicación». Revista de Humanidades, 8: 271-296.

IBARRA, A. (1998). «La agenda televisiva infantil en Guadalajara». Comunicación y Sociedad, 32: 135-168.

INESTROSA, S. (2000). «La agenda noticiosa de la prensa escrita y las iglesias en México». Anuario de Investigación de la Comunicación CONEICC, 6: 29-42.

LOZANO, J.C. (1990). "Un semestre Salinista en los MMC norteamericanos: análisis de contenido». Revista Mexicana de Comunicación, 11 (mayo-junio): 32-36.

- (1993). «Fuentes y versiones gubernamentales en la prensa mexicana: la cobertura del ataque a la aduana de Nuevo Laredo». Comunicación y Sociedad, 18/19: 179-206.

- (1994). «Tema e fontes na informação nacional em La Jornada, El Norte e El Porvenir: uma análise da sociologia da organização dos meios». Comunicaçao \& Sociedade, 13 (22): 61-83.

- (1995/96). «Oferta y recepción de televisión extranjera en México». Comunicación y Sociedad, 25/26: 259-284. 
- (1998). «Programación en la televisión aérea y de paga en el área metropolitana de Monterrey, N.L.». En: M. Ceballos [ed.]. Monterrey 400: estudios de historia y sociedad (p. 301-320). Monterrey: Universidad Autónoma de Nuevo León.

- (2000). «Información internacional y prensa latinoamericana». Diálogos de la Comunicación, 57: 49-60.

- (2000). "Oferta y consumo de contenidos televisivos transnacionales en México». Estudios sobre las Culturas Contemporáneas, 6 (12) (2. a época): 111-126.

- (2001). «Espectacularización en la cobertura informativa de las elecciones mexicanas a la presidencia». Comunicación y Sociedad, 14 (1): 29-49. Universidad de Navarra (Pamplona).

LozAnO, J.C.; De la Fuente, Y.; GarZA, R.; TreviÑo, J. (1997). «Estereotipos demográficos, ocupacionales y raciales en las películas de estreno de los videoclubes de Monterrey, Nuevo León». Revista de Humanidades, 3: 109-132.

Lozano, J.C.; De la Fuente, Y.; GarZa, R.; Treviño, J. (1999). «Imágenes y estereotipos en las películas de estreno de los videoclubes mexicanos». Revista Brasileira de Ciencias da Comunicação Intercom, 22 (2): 55-74.

Lozano, J.C.; García, H.; López, G.; Medina, J.; Mendé, M.B.; Smith, C.; Solís, M. E. (2001). «Espectacularización de las elecciones presidenciales». Revista Mexicana de Comunicación, 14 (71): 34-37.

Lozano, J.C.; Gómez, E.; Matiasich, A.; Alfonso, A.; Becerra, M.; MachadoSilveira, A.C.; Elizondo, M.; Marroquín, J.; Delgado-Aquino, L.; MartíneZ, F.J. (2000). «International news in the Latin American Press». En: MALEK, A. y KAVOORI, A. P. [eds.]. The global dynamics of news: studies in international news coverage and news agendas. P. 75-93. Stamford (Connecticut, Estados Unidos): Ablex Publishing Corporation.

LOZANO, J.C.; GARCÍA NÚNEZZ DE CÁCERES, J. (1995). «Oferta de programación audiovisual extranjera en la televisión aérea de Monterrey, Nuevo León». En: Crovi DruetTa, Delia [coord.]. Desarrollo de las industrias audiovisuales en México y Canadá. México: Facultad de Ciencias Políticas y Sociales, UNAM.

OrellanA, L. (1997). "Necrofilia visual: análisis de contenido y de receptores de La Opinión de la Tarde». Anuario de Investigación de la Comunicación CONEICC, 4: 127-150.

OrOZCO, Guillermo; Viveros, Frank (1996). «La oferta de televisión y su percepción por jóvenes de la Ciudad de México». Anuario de Investigación de la Comunicación CONEICC, 3. México: Consejo Nacional para la Enseñanza y la Investigación de las Ciencias de la Comunicación.

Pérez, M. (1997). «El caso de los balseros cubanos desde la óptica del periódico El Norte de Monterrey». Revista de Humanidades, 2: 191-212.

QUINTERO, C.; LÓPEZ, J. R. (1999). «Estereotipos de la mujer en las telenovelas mexicanas». Revista de Humanidades, 6: 245-268.

SÁNCHEZ RuIZ, E. (1995). «La agenda televisiva en Guadalajara». En: CROvi DrueTTA, Delia [coord.]. Desarrollo de las industrias audiovisuales en México y Canadá. México: Facultad de Ciencias Políticas y Sociales, UNAM.

- (1996). «Flujos globales, nacionales y regionales de programación televisiva: el caso de México». Comunicación y Sociedad, 27: 43-88.

Santa CruZ, A.; Erazo, V. (1981). Compropolitan. México: ILET; Editorial Nueva Imagen.

ToussaINT, F. (2000). «Las campañas electorales del 2000 en televisión: el caso mexicano». Revista Mexicana de Ciencias Políticas y Sociales, 44 (180): 39-56. 
Trejo Delarbre, R. (1991a). «El proceso electoral de 1988». En: Arredondo, P; Fregoso, G. y Trejo DelarbRE, R. [eds.]. Asi se calló el sistema: comunicación y elecciones en 1988. Guadalajara: Universidad de Guadalajara.

- (1991b). "Campañas y elecciones en la prensa de la ciudad de México». En: Arredondo, P.; Fregoso, G. y Trejo Delarbre, R. [eds.]. Así se calló el sistema: comunicación y elecciones en 1988. Guadalajara: Universidad de Guadalajara.

José Carlos Lozano obtuvo su grado de Maestría en Comunicación en la Universidad de Leicester, Inglaterra, y el Doctorado (Ph.D.) en Comunicación por la Universidad de Texas en Austin, Estados Unidos. Es actualmente titular de la Cátedra de Investigación en Medios Audiovisuales y Globalización en América del Norte y Coordinador de la Maestría en Comunicación en el Tecnológico de Monterrey (Monterrey, México). 